the inclination to be 144057 . The other elements are totally discordant, the orbit being hyperbolic, and the perihelion-distance 2.175 . The series of observations extends through three months, a fact which also seems adverse to the hypothesis of identity; but $I$ have here no means of reference to the observations themselves, since Hensel gives only a table of residuals between the observed positions and those deduced from Bessel's

Cordoba 1880, Febr. 26. provisional elements. A very slight examination would show whether the observed geocentric path was compatible with such an orbit as the present one. Finally Pingre mentions a comet with a tail $3 \mathrm{C}^{0} \mathrm{long}$, observed by Apian and Gemma Frisius in January 1538, and there are several records of earlier comets which favor the hypothesis of identity with the recent one and a gradually increasing period.

\title{
Untersuchungen über den grossen südlichen Cometen von 1880 .
}

Circular der Kaiserlichen Akademie der Wissenschaften in Wien. Von Prof. Dr. E. Weiss.

Der Umstand, dass die von Ralph Copeland aus den ersten, allerdings nur genäherten Beobachtungen, welche die Cap-Sternwarte von dem grossen südlichen Cometen mittbeilt, abgeleiteter Elemente eine entfernte Aehnlichkeit mit den Elementen des grossen Märzcometen von 1843 aufweisen, wie dies auch der Herr Berechner bemerkt, verbunden mit dem Umstande, dass der jetzige Comet in seiner ganzen Erscheinung eine iiberraschende Aehnlichkeit mit dem ebengenannten $\mathrm{Co}_{9}$ meten zeigte, veranlasste mich zu untersuchen, ob der Lauf des neuen Cometen nicht etwa mit den Elementen des damaligen darstellbar sei. Zu diesem Zwecke reducirte ich die letzten Elemente von Hubbard auf das mittlere Aequinoctium 1880.0, vernachlässigte die Excentricität, die bei dieser vorläufigen Untersuchung nicht in Betracht kommen kann, und legte den Periheldurchgang auf Jan. 27.6 mittl. Berliner Zeit, mit anderen Worten, ich ging von den Elementen aus:

$$
\begin{aligned}
& T .=1880 \text { Januar } 27.600 \text { mittl. Berl. Zeit. } \\
& x=84^{\circ} 20^{\circ} 42^{\prime \prime} \\
& { }^{\circ}=14559 \text { mittl. Aeq. } 1889.9 \\
& i=1441939 \\
& \log q=7.743377 \text {. }
\end{aligned}
$$

\begin{tabular}{|c|c|c|c|}
\hline & 9 & ar mittl. & Berl. Zeit \\
\hline ebr. & 10 & $0^{\text {hl }} 5^{\mathrm{m} l}$ & $-33^{\circ} 34$ \\
\hline & 11 & 021.8 & 3321 \\
\hline & 12 & 037.8 & $33 \quad 1$ \\
\hline & 13 & 053.1 & $32 \quad 33$ \\
\hline & 14 & 17.8 & 3158 \\
\hline & 15 & 121.4 & 3120 \\
\hline
\end{tabular}

\begin{tabular}{|c|c|c|c|c|}
\hline Cap MI & an : & Time & 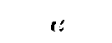 & $g$ \\
\hline Febr. & 10) & $91 / 4$ & in $4^{\mathrm{m}}$ & $-33^{\circ} 40$ \\
\hline$"$ & 11 & $83 / 4$ & 021 & 3331 \\
\hline$\eta$ & 12 & 9 & 037 & 3311 \\
\hline$"$ & 13 & $81 / 2$ & 052 & 3244 \\
\hline 3 & 14 & $83 / 4$ & 16 & 3210 \\
\hline " & 15 & $81 / 2$ & 120 & -3134 \\
\hline
\end{tabular}

Damit gestaltet sich der Lauf des Cometen zwischen Februar 10-15:

wäbrend die Beobachtungen rom Cap lauten:
Nach diesen Resultaten kann es wohl kaum einem Zweifel unterliegen, dass die beiden Himmelskörper identisch sind. Geht man übrigens mit einer Cimlaufs. zeit von 36.9 Jahren um 21 Umläufe zurück, so stösst man auf den grossen Cometen von 1106, dessen Identität mit dem Märzcometen von 1843 schon damals von vielen Seiten vermuthet wurde.

Hält man nun an der Identität der beiden Cometen fest, so müsste der Comet jetzt in unseren Gegenden sichtbar sein, falls er nicht bereits zu lichtschwach geworden ist; sein Lauf in den nächsten Wochen wäre nämlich der folgende:

1880 12 Uhr mittl. Berl. Zeit

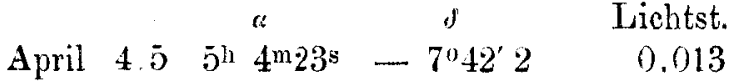

$$
\begin{aligned}
& \Rightarrow \quad 8.5 \quad 11 \quad 16 \quad 659.5 \\
& \text { "12.5 } 1746 \quad 621.9 \\
& \begin{array}{llll}
76.5 & 2359 & 5 & 48.8
\end{array} \\
& \text { "20.5 } 2956 \quad 519.9 \\
& \text { "24.5 } 53541-454.6 \quad 0.006
\end{aligned}
$$

Der Lichtstärke liegt als Finheit die vom 10. Februar zu Grunde.

\section{$\mathrm{N}$ a e h t r a g.}

Seit dem Erscheinen des Circulares habe ich noch die genäherte Beobachtung des Cometen von Gould:

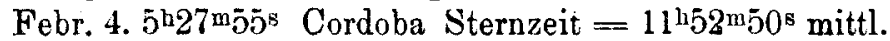
Berl. Zt.: $\alpha=22^{\mathrm{h}} 24^{\mathrm{m}} 10^{\mathrm{s}} \cdot g=-31^{\circ} 29^{\prime} 1$ mit den 
oben angeführten Elementen von Hubbard verglichen. Sie ergeben für diese Zeit $a=22^{\mathrm{h}} 23^{\mathrm{m}} 36^{\mathrm{s}} \delta=-31^{0} 17^{\prime} 8$, also dem beobachteton Orte wieder so bahe, dass sie eine weitere Bestätigung der Identität dieses Cometen mit dem von 1843 gewähren.

Zur Beurtheilung der Frage, ob der Comet noch hinreichend lichtstark sein dürfte, um ihn in unseren Breiten aufzufinden, ist die Notiz von D. Gill von Wichtigkeit, dass er am 23. Februar (also 2 Tage vor Vollmond) des hellen Mondscheines wegen keine Spur von ihm sehen konnte. Damals hatte der Comet eine Helligkeit von 0.22 , wenn man die vom 10. Februar als Einheit beibehält, war also beiläufig $17 \mathrm{mal}$ heller als am 4. April. Bedenkt man nun, dass allen unseren bisherigen Erfahrungen zufolge Cometen mit kleiner Periheldistanz viel rascher an Helligkeit abnehmen, als die gebräuchiiche Formel ergiebt, so ist die Hoffnung. ihn jetzt noch auf der nördlichen Halbkugel zu sehen, wohl eine sehr geringe, wie denn auch in der That von einer Auffindung desselben bisher nichts verlautete.

Nachdem, wie ich glaube, die Identität des Cometen mit dem Märzcometen von 1843 nicht mehr bezweifelt werden kann, bietet von den verschiedenen, seinerzeit für diesen Cometen angegobenen Umlaufszeiten dic von Boguslawski vermuthete mit $147 \frac{\mathrm{d}}{\mathrm{d}}$. h. sehr nahe gleich $4 \times 36.9$ Jahren das meiste Interesse dar. Er wurde dadurch auf sie geführt, dass er durch Zurückrecbnen mit derselben fast bei jedem Umlaufe bis zurïck zum Tahre 371 v. Chr. G. auf Cometen stiess, bei denen sich die freilich meist sehr unvollkommenen und dürftigen uns überlieferten Nachrichten ganz gut mit dem Cometen von 1843 vereinigen lassen. (Verhandlungen der schlesischen Gesellschaft für vaterländische Cultur 1845, S. 87). Die viermal kürzere Umlaufszeit fügt aber den schon von Boguslawski angegebenen, nur noch wenige Cometen-Erscheinungen hinzu, welche mit einiger $\mathrm{Wabr}$ scheinlichkeit auf den unsrigen zurïckgeführt werden können, wie mir eine Durchsicht von Pingré's Cométographie und Williams Observations of Comets, extracted from the Chinese Annals zeigte. Dies bei einem so auffallenden Himmelskörper für den ersten Augenblick überraschende Resultat ist aber nur eine natürliche Folge seiner aussergewöbnlichen Bahn. Denn nach derselben kann er auf der nördlichen Halbkugel, die für frühere Jahrbunderte allein in Betracht zu ziehen ist, nur dann auf kurze Zeit dem freien Auge sichtbar werden, wenn er sein Perihel im Februar und März oder October und November passirt. Bei einer Umlaufszeit von 36 Jahren 11 Monaten bleibt er daher nach jeder beobachteten Erscheinung, die zwei bis drei folgenden unsichtbar.

Von den oben erwähnten, neu hinzutretenden Erscheinungen des vorliegenden Cometen will ich vorläufig nur auf zwei Tagbeobachtungen von Gestirnen aus friiheren Jahrhunderten aufmerksam macben, von denen ibm wenigstens die eine wohl zweifellos angehört. Es sind nach Pingrè's Cométographie die nachstehenden:

1179. Vers la sixième heure du jour le $1^{\text {cr }}$ Août, on vit une étoile près du soleil $(I, 395)$.

1511. Cardan dit avoir vu en 1511 à Milan, en plein jour et par un ciel fort serein, une étoile extrêmement éclatante (I, 48:3).

Die erste Beobachtung liegt bis auf wenige Wochen genau gerade um 19 Umläufe zurïck, und kann um so eher auf den Cometen gedeutet werden, als er ja auch im Jahre 1843 zur Zeit des Perihels sehr bequem mit freiem Auge gesehen wurde. Bei der zweiten Beobachtung ist weder die Zeit, noch auch die Lage des Gestirnes gegen die Sonne angeführt. Fs kann daher auch wohl eine Tagbeobachtung der Venus gewesen sein, doch bleibt es immerhin beachtenswerth, dass das $\mathrm{J}$ ahr 1511 um 10 Umläufe zurückliegt.

Pingré führt noch eine dritte Tagbeobachtung an, welche ebenfalls unserem Cometen angehören künnte, wenn man sich erlauben dürfte, die Jahreszahl 1364 statt 1363 zu lesen, was aber obne eingehendere Discussion nicht zu entscheiden ist. Die Stelle lautet (I, 440):

La veille de la Trinité (27. Mai) arant midi, vers la troisième hour du jour, on vit à Paris une étoile très-petite vers le lieu du ciel où le soleil est $\dot{a}$ midi; on la distingua durant quelques jours.

Pingré hält dies sonderbarer Weise für Beobachtungen der Venus, wenige Tage nach ihrer unteren Conjunction mit der Sonne.

Währing, den 6 April 1880.

Edmund Weiss.

Entdeckung eines Cometen.

Telegraphische Depesche der K. Akademie der W. in Wien an den Herausgeber.

Comet Schäberle, 6. April $11^{\mathrm{h}}$ p. m; $\quad a=7^{\mathrm{h}} 20^{\mathrm{m}}, \quad d=+84^{\circ} 25^{\prime} ;-3^{\mathrm{m}}-48^{\prime} ;$ tail, $3^{\prime} .^{*}$ )

*) Der Wortlaut der vorstehenden, theilweise verstimmelten und nicht in der gebräuchlichen Form abgefassten Depesche iässt uicht erkennen, ab die Rectascension in Zeit oder Bogen gegeben ist. Der Ort der Entdeckung ist vermuthlich Ann Arbor. Die Red. 